\title{
Endocorona como alternativa para rehabilitar dientes endodonciados. Reporte de caso
}

\author{
Endocrown as an alternative to rehabilitate endodontic teeth. Case report
}

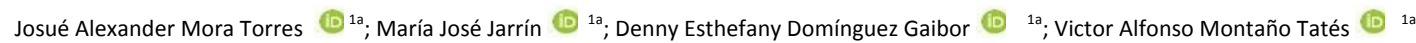

\section{RESUMEN}

El objetivo de este estudio es devolver mediante el plano oclusal la estética y funcionalidad de una pieza tratada endondónticamente en el sector posterior a través de una endocorona. El siguiente caso se trata de un paciente masculino de 27 años de edad, sin antecedentes médicos relevantes quién asistió a la clínica odontológica SERODU. Al examen radiográfico se observó los conductos endodonciados, que estaban protegidos por una base cavitaria y obturación provisional. Al examen intraoral se pudo observar que la pieza $N^{\circ} 46$ perdió la pared distal y parte de la palatina por lo que se decidió llevar acabo la rehabilitación con una endocorona. Las endocoronas ofrecen varias ventajas como el enfoque mínimamente invasivo y la conservación de la estructura dental evitando la colocación de pernos o postes y al tener un buen sellado hermético a nivel coronal se impide una posible recontaminación del tratamiento endodóntico.

Palabras clave: Adhesión; Cerómero; Endocorona; Endodoncia; Prostodoncia. (Fuente: DeC SBIREME)

\section{ABSTRACT}

The objective of this study is to restore, through the occlusal plane, the aesthetics and functionality of a piece treated endodontically in the posterior sector through an endo-crown. The following case is a 27 -year-old male patient with no relevant medical history who attended the SERODU dental clinic. The radiographic examination revealed the endodontic canals, which were protected by a cavity base and provisional filling. The intraoral examination revealed that tooth number 46 lost the distal wall and part of the palatine, so it was decided to carry out the rehabilitation with an endo-crown, as it has a good hermetic seal at the coronal level, it prevents possible re-contamination of the treatment endodontic resulting in a conservative and feasible alternative.

Key words: Adhesion; Ceromer; Endocrown; Endodontics; Prosthodontics. (Source: MeSH NLM)

1. Universidad Tecnológica del Ecuador(UTE). Quito, Ecuador.

a. Odontólogo.

\section{Correspondencia:}

María José Jarrín

Dirección: Calle Bolívar 6-38 y González Suárez. Quito, Ecuador.

Correo electrónico: majp_1995@outlook.com
Este es un artículo de acceso abierto distribuido bajo la licencia Creative Commons Atribución 4.0 Internacional (CC BY 4.0)

https://creativecommons.org/licenses/by/4.0/d

Citar como: Mora Torres JA; Jarrín MJ; Domínguez Gaibor DE; Montaño Tatés VA. Endocorona como alternativa para rehabilitar dientes endodonciados. Reporte de caso. KIRU. 2021 Jul-Sep; $18(3)$ : $172 \quad-177$. https://doi.org/10.24265/kiru.2021.v18n3.06 


\section{INTRODUCCIÓN}

La rehabilitación en el sector posterior desde hace mucho tiempo ha sido un reto para el odontólogo por factores biológicos como son el pronóstico endodóntico y periodontal, evaluación del riesgo de caries individual, anatomía radicular, remanente tisular coronario, también existe factores funcionales como el género, hábitos parafuncionales, ubicación del diente en la arcada dentaria y el rol del mismo en el tratamiento global, todos estos enunciados deben ser considerados antes de definir el tratamiento a realizar. Por lo tanto, en algunas situaciones clínicas, la decisión surge de un enfoque multi y/o interdisciplinario ${ }^{(1)}$.

Para muchos autores el uso de núcleos de fibra de vidrio ha sido una de las opciones restaurativas más usadas para tratar dientes endodonciados, pero a través del tiempo se ha demostrado que este tipo de rehabilitación según Biacchi produce retención de la corona protésica y debilitamiento del remanente coronal (2)

La propuesta de las endocrowns es proteger la estructura dental existente, preservándola y aprovechando al máximo las paredes de la cámara pulpar, evitando el desgaste del conducto radicular, fomentando integridad estructural, la carga funcional y la estética (3). Aparte que da una elección clínica beneficiosa con relación al sellado periférico, sirve en el tratamiento de dientes con canales radiculares calcificados, cortos o dilacerados ${ }^{(4)}$.

El diseño dental asistido por computadora / fabricación asistida por computadora (CAD/CAM) en la actualidad es el accesorio más utilizado para la fabricación de las endocrowns ${ }^{(5)}$, las endocrowns fabricadas bajo este tipo de sistemas manifiestan un contorno anatómico, tiempos cortos de trabajo, estructuras de superficie superiores y compatibilidad con la morfología natural de un diente natural, en comparación con aquellas que son realizadas mediante técnicas convencionales de laboratorio ${ }^{(6)}$.

Se ha encontrado que una buena adaptación es crítica para la longevidad de una corona un ajuste marginal deficiente puede conducir a caries secundaria, microfiltración, decoloración marginal y disolución de cemento que en el CAD CAM es más exacto a diferencia de las coronas convencionales de laboratorio, obteniendo mejores resultados en CAD/CAM ${ }^{(7)}$.

No existe un material específico para la elaboración ideal de las endocoronas, todos los materiales tiene sus ventajas y desventajas, los más utilizados son las cerámicas de discilicatos o los feldespatos y los cerómeros con las resinas nanocerámicas ${ }^{(8)}$.

Los cerómeros por ejemplo tienen un módulo de elasticidad (20-40Gps) parecido al de la dentina (20 Gps), distribuyen mejor las cargas disminuyendo considerablemente el índice de fractura, pero en cambio en el sellado marginal según Sevimli las porcelanas poseen mejor pronóstico, una mejor estética y no se manchan, pero son materiales rígidos que no distribuyen bien las cargas oclusales aumentando el índice de fracturas graves que imposibilitan que la pieza dental sea nuevamente rehabilitable ${ }^{(8)}$.

Desde la aparición de los sistemas adhesivos, la endocorona ha sido tomada en cuenta como una alternativa para la rehabilitación de dientes endodonciados, sirven para los órganos dentales posteriores, particularmente aquellos con coronas clínicamente bajas, conductos radiculares calcificados o canales angostos ${ }^{(9)}$, según lo establecido por Biacchi, las endocoronas adquieren función, estética y preservan la integridad biomecánica de los dientes posteriores no vitales ${ }^{(19)}$.

Entre los beneficios de la endocorona tenemos que facilita los pasos de toma de impresión, salvaguarda el periodonto, preserva la estructura sólida del remanente coronal ${ }^{(10)}$ y las cargas oclusales se distribuyen mejor sobre la articulación cervical (compresión) y las paredes axiales (fuerza de corte), moderando así la carga sobre el piso pulpar ${ }^{(11)}$.

\section{REPORTE DEL CASO}

Paciente masculino de 27 años, sin antecedentes relevantes, asintomático, refiere que "desea rehabilitarse su muela". Se evaluó clínica y radiográficamente.

Diagnóstico: Diente endodónticamente tratado y periodonto sano.

Plan de tratamiento: Se consideró que la pieza dentaria \# 46, tendría un pronóstico favorable, si se restauraba con una una endocorona 
sin la necesidad de colocar perno muñón colado o prefabricado, aplicando así un tratamiento protésico conservador, estético y funcional.

\section{Ejecución del Tratamiento}

En la primera cita se realizó la valoración clínica y radiográfica del diente a tratar (figura $1 \mathrm{~A}$ ), con el cual se decidió realizar levantamiento del margen o Build up a nivel distal del diente \#46, donde había pérdida de estructura, utilizando un sistema de resina tipo BULK (figura 1B). Se realizó una delicada retracción de tejidos blandos y aislamiento absoluto del campo operatorio con el objetivo de obtener una línea de terminación supragingival evitando un posible problema periodontal a futuro.

El tallado se inició con una fresa de fisura para romper el punto de contacto y evitando el desgarre de la silicona de adición al momento de retirarla, cabe recalcar que las piezas adyacentes fueron protegidas con una banda metálica (figura 1C). Para la conformación de la terminación y de las paredes tanto internas como externas de la pieza a tratar, se utilizó una fresa tronco cónica punta redonda ISO $197^{\mathrm{a}}$, obteniendo una conformación a nivel de la cámara pulpar con una divergencia de al menos $7^{\circ}$ hacia oclusal. La línea de terminación externa fue tipo chamfer, obteniendo una altura final $5 \mathrm{~mm}$ ya que presentaba suficiente espacio para generar una retención estable (figura 1D).

Para la toma de impresión se utilizó silicona de adición o polivinil siloxanos (pvs), con técnica dos pasos (figura 1E), para el registro intermaxilar se usó silicona de condensación y para el modelo antagonista alginato. Para la provisionalización del diente \# 46 se utilizó un material de obturación fluido y fotopolimerizable (CLIP), las razones principales del uso del mismo, fue por el corto tiempo de utilización, la facilidad de su retiro, así la cavidad permanece sin cambios por la eliminación de Clip, y no hay efecto adverso sobre el ajuste de la restauración definitiva.

La toma de color se la realizó con el colorímetro Vita Classic, obteniendo un color A2. Para la elección del material se tomó en cuenta el antagonista y la fuerza de mordida originada por cada material,. El cerómero fue el material de elección debido a que posee una adecuada resistencia frente a fuerzas masticatorias por su módulo de elasticidad permitiendo liberar tensión de una manera óptima y acompañando así a las características de flexión natural del diente. Se envió al laboratorio de prótesis, especificando el color, el tipo de material, las impresiones definitivas, el modelo antagonista, registro intermaxilar y un registro fotográfico para que el técnico tenga una visión más amplia; incluyendo además datos del paciente como la edad y el sexo.

Una vez recibida la restauración protésica, se verificó su adaptación sobre el modelo enviado por el laboratorio (figura 1F), posteriormente se desinfectó utilizando alcohol isopropil; se realizó una prueba en boca verificando con una sonda periodontal su correcta adaptación marginal y adecuado asentamiento sobre la estructura dental, además se verificó contactos oclusales estables y parámetros estéticos.

Para la cementación primero se preparó la restauración arenándola con oxido de aluminio de 50 micras, por 12 segundos, para el acondicionamiento de la endocorona se grabó con ácido fosfórico al 35\% Etchant Gel S de la casa comercial COLTENE por 20 segundos (figura 1G), se realizó el lavado y secado por 40 segundos, se aplicó el sistema adhesivo Tetric $\mathrm{N}$-bond Universal de la IVOCLAR (figura $1 \mathrm{H}$ ), se hizo la evaporación con aire por 20 segundos.

Una vez preparada la restauración se procedió a desinfectar el diente con clorhexidina al $2 \%$. Se hizo el grabado total por 5 segundos con ácido ortofosfórico al $35 \%$ Etchant Gel S (COLTENE), lavado por 30 segundos (figura 1I), secado con papel absorbente, aplicación de adhesivo Tetric N-bond Universal IVOCLAR. Evaporación del solvente con aire por 20 segundos y fotopolimeración (figura $1 \mathrm{~J}$ ).

Para la cementación se utilizó cemento resinoso dual Color A3 Allcem FGM con una fotopolimeración inicial de 5 segundos a nivel oclusal, con lámpara de Polimerizar "Woodpecker", verificando previamente su correcto asentamiento. Se eliminó el material excedente utilizando hilo dental a nivel interproximal y marginal respectivamente, para finalmente realizar una fotopolimeración de 40 segundos por cada cara del diente, previamente se colocó una capa de glicerina gel para evitar la capa inhibida e oxígeno (figura $1 \mathrm{~K}$ ). Una vez terminado el protocolo de cementación (figura $1 \mathrm{~L})$, se realizó control oclusal utilizando papel articular verificando estabilidad en los puntos de contacto, posteriormente acabado y pulido de la interfase restauración-diente. Se informó al paciente de las citas de mantenimiento. Se le recomendó que continúe con un tratamiento de ortodoncia y la colocación de implantes o prótesis fija, pero refirió que por motivos económicos lo realizaría posteriormente. 


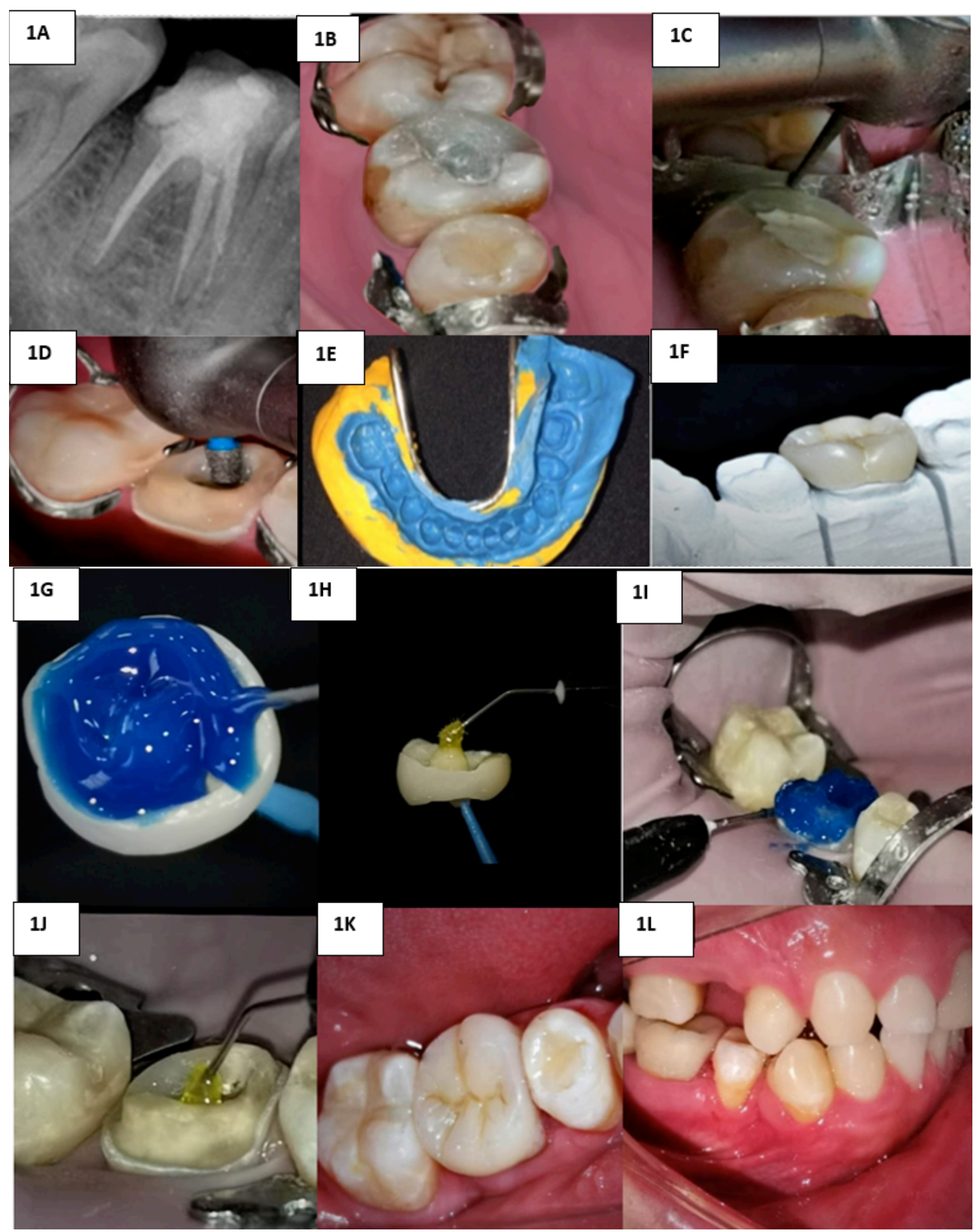

1A. Radiografía periapical de la pieza \#46. 1B. Levantamiento del margen o Build up a nivel distal.1C. Inicio de tallado con una fresa de fisura para romper el punto de contacto. 1D. Conformación de la terminación y de las paredes tanto internas como externas con una fresa tronco cónica punta redonda ISO 197a.1E. Impresión con silicona de adición con técnica dos pasos. 1F. Adaptación sobre el modelo enviado por el laboratorio. 1G. Acondicionamiento de la endocorona. 1H. Aplicación de adhesivo Tetric N-bond Universal IVOCLAR en la endocorona. 1I. Acondicionamiento de la pieza dental. 1J. Aplicación de adhesivo Tetric N-bond Universal IVOCLAR de la pieza dental.1K.Cementación de la endocorona. 1L. Control oclusal. 


\section{DISCUSIÓN}

En el presente caso se observó que la pared distal se encontraba a nivel del margen cervical y las demás paredes se encontraban a nivel oclusal, según Borgia, los niveles del margen cervical no deben tener una pendiente de más de $60^{\circ}{ }^{(9)}$, Sevimli, G en su artículo opina lo mismo ya que se puede producir un efecto en escalera ocasionando un desprendimiento de la endocorona ${ }^{(8)}$, al no poseer pared distal el desgaste de las paredes hubiera sido excesiva para conseguir los parámetros recomendados, es por eso que se buscó otra alternativa.

Rocca sugiere que para que aumente la probabilidad de éxito de las endocrown es necesario una conservación de la mayor parte del remanente coronal, es por eso que al no existir un margen óptimo, se lo puede elevar con incrementos de resina convencional, los beneficios que conlleva esto son una geometría ideal, reubicación de la línea de terminación de forma supragingival y espacio interoclusal adecuado $^{(12)}$, esto se tomó en consideración y se realizó una elevación de la pared distal con resina compuesta bulk fill de la casa comercial $3 \mathrm{M}$, se consiguió una estructura óptima para el inicio del tallado y se preservó el tejido dental.

Muchos autores prefieren el uso de discilicatos y feldespatos como material para la fabricación de las endocoronas, tienen muchos beneficios ya sea por su resistencia, estética, y el sellado marginal óptimo que se obtiene ${ }^{(8)}$, pero a lo que respecta los índices de fractura el cerómero tiene un mejor pronóstico ya que distribuye mejor las cargas oclusales $^{(13)}$. El-Damanhoury HM hizo un estudio in vitro donde comparo dichos materiales, se descubrió que los discilicatos y feldespatos poseían un tipo de fractura que imposibilitaban la rehabilitación protésica a diferencia de los cerómeros ${ }^{(14)}$, el material a elección fue una resina nanocerámica por tener una pared reconstruida con resina se estimaba que el uso de porcelanas podría perjudicar la longevidad del tratamiento, lo que se obtuvo fue un buen sellado marginal, óptima adaptabilidad y una excelente estética $^{(3)}$.

En cuanto a la longevidad no se puede asegurar ya que necesitamos controles periódicos a largo plazo, entre los estudios clínicos longitudinales más relevantes que se obtuvieron, fueron los de Otto y Mörmann, que de 25 endocoronas se presentó el éxito de $90.5 \%$ en molares durante 12 años ${ }^{(15)}$ y el de Bindl, que tuvo un éxito del $80 \%$ en 70 molares durante 8 años, dichos autores también hacen alusión que en los molares existe un mejor pronóstico en comparación a los premolares donde hay mayor número de fracasos ${ }^{(16)}$.

La conformación de la cavidad fue realizada con la toma de varios artículos, según Hayes, la conformación de la cámara pulpar debe ser 5 $\mathrm{mm}$ de diámetro por $5 \mathrm{~mm}$ de profundidad ${ }^{(4)}$, para Fages las paredes no deben exceder una divergencia que vaya de los 7 a 10 grados $^{(10)}$, Lander además también nos propone el uso de una línea de terminación chaflán supragingival (17), lo único que no se tomó en consideración fue la disminución oclusal que según Fages es de $2 \mathrm{~mm}$ para porcelana ${ }^{(10)}$ y Rocca $1,5 \mathrm{~mm}$ para cerómeros ${ }^{(12)}$, el motivo fue que durante la etapa clínica y de laboratorio se observó que no se poseía un espacio óptimo para el material a utilizar y no cumplían con las expectativas estéticas y funcionales esperadas, es por eso que se redujo $3 \mathrm{~mm}$ en oclusal consiguiendo así lo propuesto.

No existen tantos estudios clínicos longitudinales de la endocorona ya que estos son escasos. Lander y Dietschi, presentaron un reporte de caso donde realizaron dos endocoronas e hicieron un seguimiento durante 3 años ${ }^{(18)}$; Biacchi, et al. (2013), presentaron de igual forma 1 reporte de caso de 3 años donde la endocorona tuvo éxito durante el tiempo que duro el estudio ${ }^{(19)}$.

Las endocoronas ofrecen varias ventajas como el enfoque mínimamente invasivo y la conservación de la estructura dental evitando la colocación de pernos o postes y al tener un buen sellado hermético a nivel coronal se impide una posible re-contaminación del tratamiento endodóntico.

Contribuciones de autoría: JMT, MJJ, DDG, VMT. Participaron en el diseño del trabajo, obtención de resultados, análisis e interpretación de datos, redacción del manuscrito, revisión crítica, aprobación de su versión final, aporte de pacientes o material de estudio, obtención de financiamiento y asesoría técnica.

\section{Fuente de financiamiento: Autofinanciado.}

Conflictos de interés: Declaramos que no existe ningún tipo de conflicto de intereses entre los miembros de este artículo inédito.

\section{REFERENCIAS BIBLIOGRÁFICAS}

1. Einhorn M, DuVall N, Wajdowicz M, Brewster J, Roberts $\mathrm{H}$. Preparation Ferrule Design Effect on Endocrown Failure Resistance. J Prosthodont.2019;28(1):237-242. 
2. Ghoul WE, Özcan M, Tribst JPM, Salameh Z. Fracture resistance, failure mode and stress concentration in a modified endocrown design. Biomater Investig Dent. 2020 ;7(1):110-119.

3. Tribst JP, Dal Piva AO, Madruga CF, Valera MC, Bresciani E, Bottino MA, de Melo RM. The impact of restorative material and ceramic thickness on CADICAM endocrowns. J Clin Exp Dent. 2019 Nov 1;11(11):969-977

4. Hayes A, Duvall N, Wajdowicz M, Roberts $H$. Effect of Endocrown Pulp Chamber Extension Depth on Molar Fracture Resistance. 2017;42(3):327-334.

5. Sevimli G, Cengiz S, Oruc MS. Endocrowns: review. J Istanb Univ Fac Dent 2015; 49(2):57-63.

6. Tzimas K, Tsiafitsa M, Gerasimou P, Tsitrou E. Endocrown restorations for extensively damaged posterior teeth: clinical performance of three cases. Restor Dent Endod [Internet].2018 [citado 4 de agosto de 2020];43(4). Disponible en: https://www.ncbi.nlm.nih.gov/pmc/articles/PMC62 37728/

7. Dogui $H$, Abdelmalek $F$, Amor A, Douki N. Endocrown: An Alternative Approach for Restoring Endodontically Treated Molars with Large Coronal Destruction. Case Rep Dent. 2018

8. Sevimli G, Cengiz S, Oruc MS. Endocrowns: review. J Istanb Univ Fac Dent 2015; 49(2):57-63.

9. Borgia E, Barón R, Borgia José L. Endocrown: Estudio clínico retrospectivo de una serie de pacientes, en un período de 8 a 19 años. Odontoestomatología [Internet]. 2016 [citado 2020 Oct 19] ; 18( 28 ): 48-59. Disponible en: http://www.scielo.edu.uy/scielo.php?script=sci_art text\&pid=S1688-93392016000200007\&lng=es.

10. Fages $\mathrm{M}$, Bennasar B. The Endocrown: A Different Type of All-Ceramic Reconstruction for Molars. J Can Dent Assoc. 2013; 79:d140.

11. Rao, B., Bandekar, S., Kshirsagar, S., \& Naman, S. (2017). Endocrown-A Unique Way of Retention-Case Report. Journal of Advances in Medicine and Medical Research. [Internet]. 2017[citado 2020 Oct 19];22(3):1-5. Disponible en: https://doi.org/10.9734/JAMMR/2017/32747

12. Rocca G, Krejci I. Crown and post-free adhesive restorations for endodontically treated posterior teeth: from direct composite to endocrowns. Eur J Esthet Dent.2013;8:156-79.

13. Zarone F, Sorrentino R, Apicella D, Valentino B, Ferrari M, Aversa R, Apicella A. Evaluation of the biomechanical behavior of maxillary central incisors restored by means of endocrowns compared to a natural tooth: a 3D static linear finite elements analysis. Dent Mater. 2006;22(11):1035-44.

14. El-Damanhoury HM, Haj-Ali RN, Platt JA. Fracture resistance and microleakage of endocrowns utilizing three CAD-CAM blocks. Oper Dent [Internet]. 2015[citado 2020 Oct 19];40(2):201-10. Disponible en: https //www. 10.2341/13-143-L

15. Otto $\mathrm{T}$, Mörmann $\mathrm{WH}$. Clinical performance of chairside CAD/CAM feldspathic ceramic posterior shoulder crowns and endocrowns up to 12 years. Int J Comput Dent. 2015;18(2):147-61

16. Tzimas, K., Tsiafitsa, M., Gerasimou, P., \& Tsitrou, E. (2018). Endocrown restorations for extensively damaged posterior teeth: clinical performance of three cases. Restorative dentistry \& endodontics [Internet]. 2018[citado 2020 Oct 19];43(4):38. Disponible en: https://www.ncbi.nlm.nih.gov/pmc/articles/PMC62 37728/

17. Laden G, Nuran U. Effect of Endocrown Restorations with Different CAD/CAM Materials: 3D Finite Element and Weibull Analyses. BioMed Research International. [Internet] 2017[citado 2020 Sep 16].Disponible en: https://doi.org/10.1155/2017/5638683

18. Lander E, Dietschi D. Endocrowns: A clinical report. Quintessence Int 2008;39:99-106.

19. Biacchi GR, Basting RT. Comparison of fracture strength of endocrowns and glass fiber postretained conventional crowns. Oper Dent. 2012;37(2):130-6

Josué Alexander Mora Torres

ORCID iD:@ https://orcid.org/0000-0003-0266-7004

josuealex07061996@gmail.com

María José Jarrín

ORCID iD: https://orcid.org/0000-0002-1875-1254

majp_1995@outlook.com

Denny Esthefany Domínguez Gaibor

ORCID iD: https://orcid.org/0000-0002-4903-7133

stefydg_5@hotmail.com

Victor Alfonso Montaño Tatés

ORCID iD:-10 https://orcid.org/0000-0001-7328-5834

victor18alfonso@hotmail.com

Copyright $@$ La revista. La revista Kiru es publicada por la Facultad de Odontología de la Universidad de San Martín de Porres, en Lima, Perú. 